\title{
Confocal Raman mapping of collagen cross-link and crystallinity of human dentin-enamel junction
}

\author{
Amel Slimani \\ Fares Nouioua \\ Alban Desoutter \\ Bernard Levallois \\ Frédéric J. G. Cuisinier \\ Hervé Tassery \\ Elodie Terrer \\ Hamideh Salehi
}




\title{
Confocal Raman mapping of collagen cross-link and crystallinity of human dentin-enamel junction
}

\author{
Amel Slimani, ${ }^{a}$ Fares Nouioua, ${ }^{\text {a }}$ Alban Desoutter, ${ }^{\text {a }}$ Bernard Levallois, ${ }^{\text {a }}$ Frédéric J. G. Cuisinier, ${ }^{a}$ \\ Hervé Tassery, ${ }^{\mathrm{a}, \mathrm{b}}$ Elodie Terrer, ${ }^{\mathrm{a}, \mathrm{b}}$ and Hamideh Salehi ${ }^{\mathrm{a}, *}$ \\ aUniversité Montpellier, Laboratoire de Bioingénierie et Nanosciences, Montpellier, France \\ bUniversité Aix-Marseille, Department of Restorative Dentistry, Marseille, France
}

\begin{abstract}
The separation zone between enamel and dentin [dentin-enamel junction (DEJ)] with different properties in biomechanical composition has an important role in preventing crack propagation from enamel to dentin. The understanding of the chemical structure (inorganic and organic components), physical properties, and chemical composition of the human DEJ could benefit biomimetic materials in dentistry. Spatial distribution of calcium phosphate crystallinity and the collagen crosslinks near DEJ were studied using confocal Raman microscopy and calculated by different methods. To obtain collagen crosslinking, the ratio of two peaks $1660 \mathrm{~cm}^{-1}$ over $1690 \mathrm{~cm}^{-1}$ (amide I bands) is calculated. For crystallinity, the inverse full-width at half maximum of phosphate peak at $960 \mathrm{~cm}^{-1}$, and the ratio of two Raman peaks of phosphate at $960 / 950 \mathrm{~cm}^{-1}$ is provided. In conclusion, the study of chemical and physical properties of DEJ provides many benefits in the biomaterial field to improve the synthesis of dental materials in respect to the natural properties of human teeth. Confocal Raman microscopy as a powerful tool provides the molecular structure to identify the changes along DEJ and can be expanded for other mineralized tissues. ๑ 2017 Society of Photo-Optical Instrumentation Engineers (SPIE) [DOI: 10.1117/1.JBO.22.8 .086003]
\end{abstract}

Keywords: Raman microscopy; dentin-enamel junction; collagen; mineral.

Paper 170229RR received Apr. 11, 2017; accepted for publication Jul. 24, 2017; published online Aug. 18, 2017.

\section{Introduction}

A thick dentin layer and a highly mineralized and protective enamel cap form the tooth. The enamel is acellular and highly mineralized while the dentin is a protein rich calcified tissue. ${ }^{1}$ Concerning the organic matrix, the collagen type I constitutes $90 \%$ of the organic matrix and creates a 3-D network ${ }^{2,3}$ and the collagen type III and/or IV represent about $3 \%$ of the collagen. ${ }^{4}$ The DEJ is reported as a broad transitional region between two tissues with different mechanical properties, i.e., enamel is very hard and fragile, and dentin is more elastic due to the presence of collagen. ${ }^{4,5}$ The DEJ restrains crack propagation under masticatory loading and improves tooth longevity due to its particular organic and mineral patterns. ${ }^{4-6}$ Parallel collagen fibrils interleave into the enamel mineral and combine with the fibrillar network of the inner dentin matrix. ${ }^{6,7}$ The collagen fibril is formed by bundles of crosslinked microfibrils arranged by the staggering of collagen molecules. ${ }^{8}$ The endogenous crosslinks are naturally present in the collagen structure providing covalent bonds between collagen fibrils in the extracellular matrix right after fibrillogenesis. The collagen mechanical properties depend on a highly regulated mechanism of intra- and intermolecular crosslinking ${ }^{9,10}$ that influences its thermal equilibrium and mechanical strength. ${ }^{11-13}$ The crosslinking influences the stability of collagen matrix and can slow down pathological dentin caries process by improving the enzymatic degradation resistance. Thus, the crosslinking property of the dentin collagen at the DEJ is essential to understand the inter-relation between enamel and dentin. A disorder in the collagen crosslinking can cause

*Address all correspondence to: Hamideh Salehi, E-mail: s_hamideh@yahoo .com a dysfunctional tissue, like in osteoporosis and scurvy. In dentin, altered collagen crosslinks are associated to reactionary dentin and caries. ${ }^{14}$ The collagen fibers are reinforced by the mineralization of calcium phosphate crystals. Crystallinity or degree of crystallization is related to the maturation and mechanism of growth of calcium phosphate. Deposition of calcium phosphate crystal occurs either inside the collagen fibers or between the fibers. Near the DEJ, some structures can be found, such as enamel tufts and enamel spindles. Enamel tufts are hypocalcified spaces arranged between groups of enamel rods filled with organic matrix. Enamel tufts appear longer and larger than enamel spindle and they can outspread to one-fifth of the enamel surface. Enamel spindles are small extensions of the dentinal tubules. These hypocalcified structures have clinical impacts since they allow the spread of caries. ${ }^{15}$

Different techniques have been used to investigate the complex properties of DEJ and a variety of hypotheses were proposed. An atomic force microscopy-based nanoscratching method reported an intermediate functional zone DEJ around $2 \mu \mathrm{m}$ according to the viscoelastic properties. ${ }^{16}$ Studies using nanoindentation analysis described a width of DEJ range from $10-13 \mu \mathrm{m}^{17}$ to $15-25 \mu \mathrm{m}$. $^{18}$

Confocal Raman microscopy is a label-free, nondestructive, and vibrational spectroscopic technique that enables structural and chemical mapping analysis of mineralized tissue such as teeth. ${ }^{19} \mathrm{Xu}$ et al. ${ }^{20}$ measured the DEJ width depending on the intratooth location and reported that the width of the transition zone of DEJ at the occlusal position was wider than at the cervical position. They constructed a mapping of DEJ with a special resolution limited to $1 \mu \mathrm{m}$ through a wide scan of $35 \mu \mathrm{m}$.

$1083-3668 / 2017 / \$ 25.00$ (C) 2017 SPIE 
On the contrary, our study proposes a high-resolution confocal Raman microscopy of the DEJ, not achieved up to now, with a width map of $80 \mu \mathrm{m}$ and a spatial resolution of $300 \mathrm{~nm}$.

The aim of this study is to simultaneously determine the collagen crosslink ratio $\left(1660 / 1690 \mathrm{~cm}^{-1}\right)$ and the crystallinity at the DEJ with reconstructed high-definition chemical images. Two different techniques were compared to estimate the DEJ crystallinity: ratio of phosphate Raman peaks 960/950 cm and full-width at half maximum $(\mathrm{FWHM})^{-1}$ of phosphate peak at $960 \mathrm{~cm}^{-1}$; both techniques show the same crystallinity pattern.

\section{Materials and Methods}

\subsection{Specimen Preparation}

Four extracted teeth (premolars) for orthodontic reasons were obtained from Montpellier University Hospital (ethical board authorization: DC-2014-2198) and stored in ultrapure water (Millipore) at $4^{\circ} \mathrm{C}$. Samples were cleaned with sodium bicarbonate cleaning tool (Air N Go, classic powder, Actéon, Bordeaux, France) and rinsed with ultrapure water. The teeth were sectioned longitudinally using a diamond saw to have cuts of 0.5-mm thickness (Isomet 2000, Buehler, Lake Bluff). Samples were polished using silicon carbide abrasive discs and diamond pastes $(6,1$, and $0.25 \mathrm{~mm})$ using a polishing machine $\left(\mathrm{ESCIL}^{\circledR}\right.$, Lyon, France). Finally, the samples were passed through an ultrasonic bath for 5 min. ${ }^{21}$

\subsection{Raman Data Acquisition}

A Witec confocal Raman microscopy $\alpha 300 \mathrm{R}$ (Witec, Ulm, Germany) was used to collect the Raman spectra. The confocal Raman microscopy is equipped by a frequency doubled $\mathrm{Nd}$ : YAG laser (Newport, Evry, France) with the wavelength of $532 \mathrm{~nm}$ and $\times 20$ NIKON air objective with numerical aperture of 0.46 (Nikon, Tokyo, Japan). The output laser power is $50 \mathrm{~mW}$. The spatial resolution is $300 \mathrm{~nm}$ and depth resolution is roughly $1 \mu \mathrm{m}$. The microscope is equipped with a piezodriven scan stage with a positioning accuracy of 2 to $3 \mathrm{~nm}$ horizontally and $10 \mathrm{~nm}$ vertically. The acquisition time of a single spectrum was set to $0.5 \mathrm{~s} .150 \times 150$ points per image were recorded, leading to a total of 22,500 spectra for one image. Data acquisition is performed using Image Plus 2.08 software from Witec. Using an edge filter, Raman backscattered radiations are separated from the Rayleigh scattered light. The Raman photons are transferred to the electron multiplying charge-coupled device camera EMCCD (DU 970N-BV353, Andor, Hartford). The EMCCD chip size is $1600 \times 200$ pixels, the camera controller is a 16 bit $\mathrm{A} / \mathrm{D}$ converter operating at $2.5 \mathrm{MHz}$, and the camera is cooled by a Pelletier system. The UHTS 300 spectroscopy system with a $70 \%$ throughput transmission and a grating of 600 lines $/ \mathrm{mm}$ (operating at $-60^{\circ} \mathrm{C}$ ) provide a spectral resolution of 3 to $5 \mathrm{~cm}^{-1}$.

\subsection{Data Analysis}

Witec Project software (Ulm, Germany) is applied for cosmic ray removal and baseline corrections before data analysis to reconstruct the Raman images. Sharp spikes-belong to energetic cosmic ray-typically narrower than Raman bands are removed by calculation of line slop. The baseline correction algorithm approximates the background by a polynomial to remove the slight fluorescence if present. Data processing is performed using Image Plus software and Witec Project Plus (Ulm, Germany). First, the integrated Raman intensities of the area of interest at a particular peak (e.g., $960 \mathrm{~cm}^{-1}$ ) are calculated. Using a look-up table (LUT), bright yellow hues indicate the highest integrated peak intensities, whereas orange hues indicate the lowest intensities of the area of interest.

To produce crystallinity or collagen crosslink images, a ratio of two different Raman peaks is used. For each pixel, a number with a corresponding color shows the ratio of those two peaks at that pixel. For collagen crosslink, an image is obtained by the ratio of two amide I sub-bands at $1660 \mathrm{~cm}^{-1}$ over $1690 \mathrm{~cm}^{-1} \cdot 12,22$ Crystallinity is calculated using the previous ratio method for two Raman peaks at $960 \mathrm{~cm}^{-1}$ over $950 \mathrm{~cm}^{-1}$. The Witec software provides a second method to calculate crystallinity using FWHM $^{-1}$ of phosphate peak $\left(960 \mathrm{~cm}^{-1}\right)$.

To obtain the width of DEJ, the maps of phosphate, organic, collagen crosslink, and crystallinity are compared to find out the changes pattern. The cross-sections are plotted in one graph to simplify their comparison and to mark the DEJ area.

\section{Results}

The two reference spectra of the enamel (in red) and dentin (in blue) are presented in Fig. 1. In dentin, peaks related to amides and those corresponding to the organic matrix $(\mathrm{C}-\mathrm{H}$ bands) are present. Three phosphate peaks are common in dentin and enamel: $960 \mathrm{~cm}^{-1}\left(\nu_{1} \mathrm{PO}_{4}^{3-}\right.$, first elongation mode), $1430 \mathrm{~cm}^{-1}$ $\left(\nu_{2} \mathrm{PO}_{4}^{3-}\right)$, and $585 \mathrm{~cm}^{-1}\left(\nu_{4} \mathrm{PO}_{4}^{3-}\right)$. The organic matrix peaks are absent in enamel. The assignments of Raman peaks that are used to create the reconstructed images are presented in Fig. 1.

After selection of the area of interest in a bright-field image, scan is started to acquire Raman spectra one per pixel. Figure 2(a) is a bright-field image of the DEJ: enamel is above and dentin is below. Figure 2(b) is a representative Raman image of the DEJ based on intensity of $960 \mathrm{~cm}^{-1}$ phosphate peak $\left(\nu_{1}\right.$ $\mathrm{PO}_{4}^{3-}$ ). The enamel [upper part of Fig. 2(b)] hues vary from bright yellow to orange as it approaches the dentin, where the phosphate tends to decrease. Here, the first symmetric stretching vibration of phosphate $\left(\nu_{1}\right)$ is chosen to construct a Raman map of phosphate because it is stronger than the other two peaks of bending modes of phosphate $\left(\nu_{2}, \nu_{4}\right) \cdot{ }^{23}$ In the same area, enamel spindles are described by the dark orange and black cracks, which relate to low phosphate intensities. Below the enamel [Fig. 2(b)], dentin is arranged as a series of parallel dentinal tubules that go from black to dark orange hues since it is a poor mineral tissue compared to enamel.

Figure 2(b) contains 22,500 pixels and the intensity of phosphate peak varies according to the pixel's content. Using Witec software, each pixel's phosphate content is presented with a false color. Checking LUT, phosphate peak intensity would be defined.

Figure 3(a) presents a reconstructed image based on the intensity of the phosphate Raman $\nu_{1} \mathrm{PO}_{4}^{3-}$; each pixel in the image corresponds to the intensity of the selected peak and it is represented by color differences. Enamel is located in the upper left angle and dentin is in the rest of the image. In enamel, higher phosphate intensity is noticeable (red hues) and it decreases while crossing the DEJ. Along dentinal tubules, a regular pattern of phosphate is observed. Red longitudinal areas in Fig. 3(a) represent high phosphate content and correspond to the peritubular and/or intertubular dentin, where phosphate is at its maximum. Figure 3(b) represents an image of the collagen crosslink based on the intensity of amid I sub-bands from 1660 


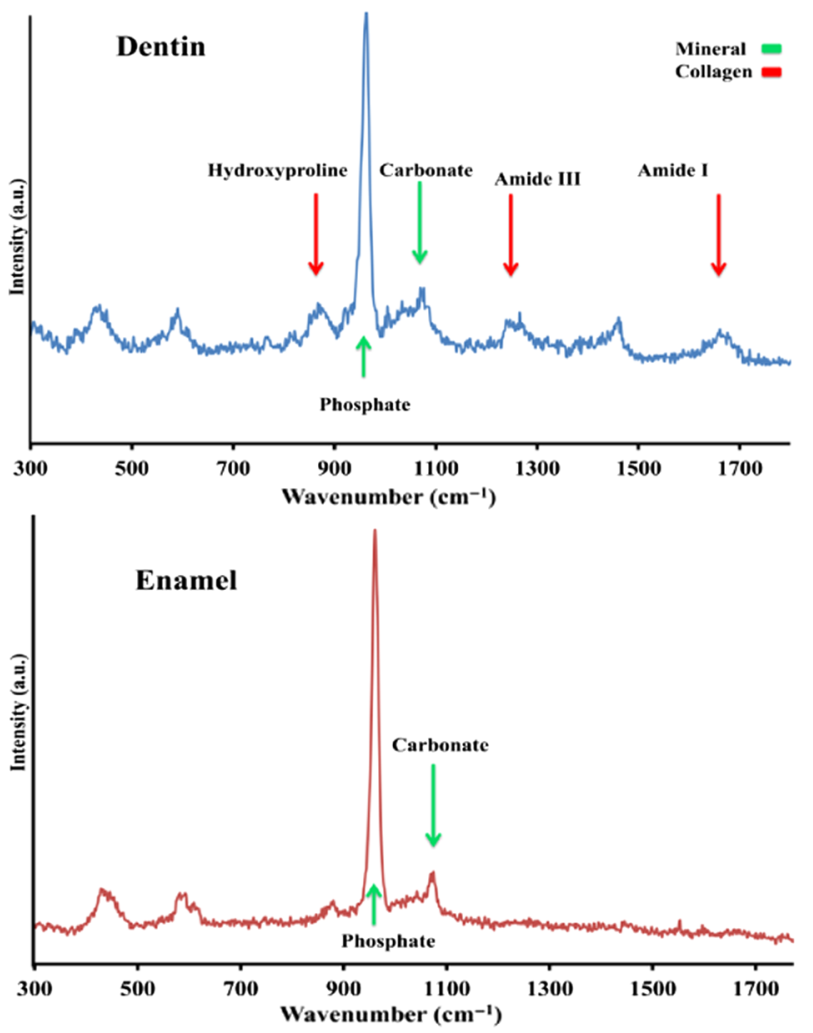

\begin{tabular}{|c|c|c|}
\hline $\begin{array}{l}\text { Wavenumbers }\left(\mathrm{cm}^{-1}\right) \text { and band } \\
\text { assignments of Raman spectra from } \\
\text { sound enamel and dentin }\end{array}$ & Enamel $\left(\mathrm{cm}^{-1}\right)$ & Dentin $\left(\mathrm{cm}^{-1}\right)$ \\
\hline $\mathrm{v}_{2}$ Phosphate $\left(\mathrm{PO}_{4}{ }^{3-}\right)$ & 433 & 432 \\
\hline $\mathrm{v}_{4}$ Phosphate $\left(\mathrm{PO}_{4}{ }^{3-}\right)$ & $(579-608)$ & $(580-610)$ \\
\hline$v_{1}$ Phosphate $\left(\mathrm{PO}_{4}{ }^{3-}\right)$ & 959 & 959 \\
\hline B type $v_{1}$ Carbonate $\left(\mathrm{CO}_{3}{ }^{2-}\right)$ & 1071 & 1069 \\
\hline A type $v_{1}$ Carbonate $\left(\mathrm{CO}_{3}{ }^{2-}\right)$ & 1103 & 1102 \\
\hline (NH) Amide III & & 1243 \\
\hline $\begin{array}{l}\text { (NH) Amide III non-polar triple helix of } \\
\text { collagen }\end{array}$ & & 1275 \\
\hline CH (deformation) & & 1450 \\
\hline Amide I $(\mathrm{C}=\mathbf{O})$ & & 1665 \\
\hline Pentosidine & & 1550 \\
\hline AGEs & & $1550-1690$ \\
\hline Phosphate to organic matrix ratio & & $960 / 1450$ \\
\hline Crystallinity & $960 / 950$ & $960 / 950$ \\
\hline Crystallinity second method & $\mathrm{FWHM}^{-1}[959]$ & $\mathrm{FWHM}^{-1}[959]$ \\
\hline Collagen cross-link ratio & & $1660 / 1690$ \\
\hline
\end{tabular}

Fig. 1 The reference spectra of dentin in blue and enamel in red are presented. The table of assignments of Raman peaks presented in enamel and dentin. The organic elements are undetectable in enamel.
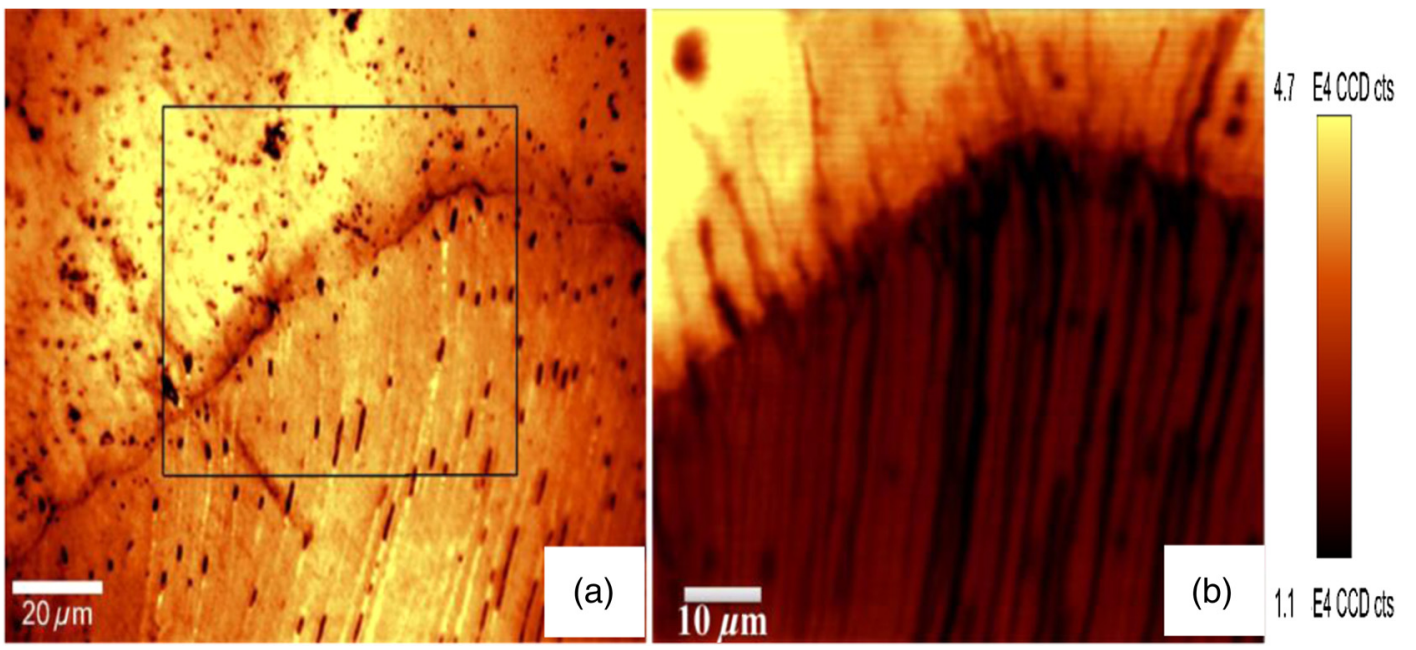

Fig. 2 DEJ of a human tooth: (a) bright-field image of the DEJ: black square indicates the scanned area to acquire Raman spectra for image (b). (b) Representative image of the DEJ based on Raman intensities of phosphate $\nu_{1} \mathrm{PO}_{4}^{3-}$ peak. Enamel spindles are hypocalcified spaces in enamel starting at the DEJ. Bright yellow hues indicate the highest integrated phosphate intensities and the dark hues the lowest respectively (see LUT on the right).

to $1690 \mathrm{~cm}^{-1}$. Gradually, collagen crosslink increases going from red (inside the dentinal tubules) to purple in enamel since there is no collagen in this tissue [Fig. 3(b)]. Clearly, collagen crosslink ratios' intensity is zero in enamel, and passing by the DEJ toward dentin, it starts to increase. High collagen crosslink inside tubules is linked to low phosphate. Along dentinal tubules, decreasing phosphate happens when collagen crosslink increases. The two crystallinity images have similar pattern to phosphate image [Fig. 3(a)] and are opposite of collagen crosslink image [Fig. 3(b)]. Crystallinity in enamel is at its maximum and decreases during the transition from the enamel to dentin. The two methods that are used to determine crystallinity show a similar pattern with details given in Fig. 5.

Reconstructed Raman images of the DEJ are presented in Fig. 4. The phosphate $\left(960 \mathrm{~cm}^{-1}\right)$ image is shown in Fig. 4(a). 


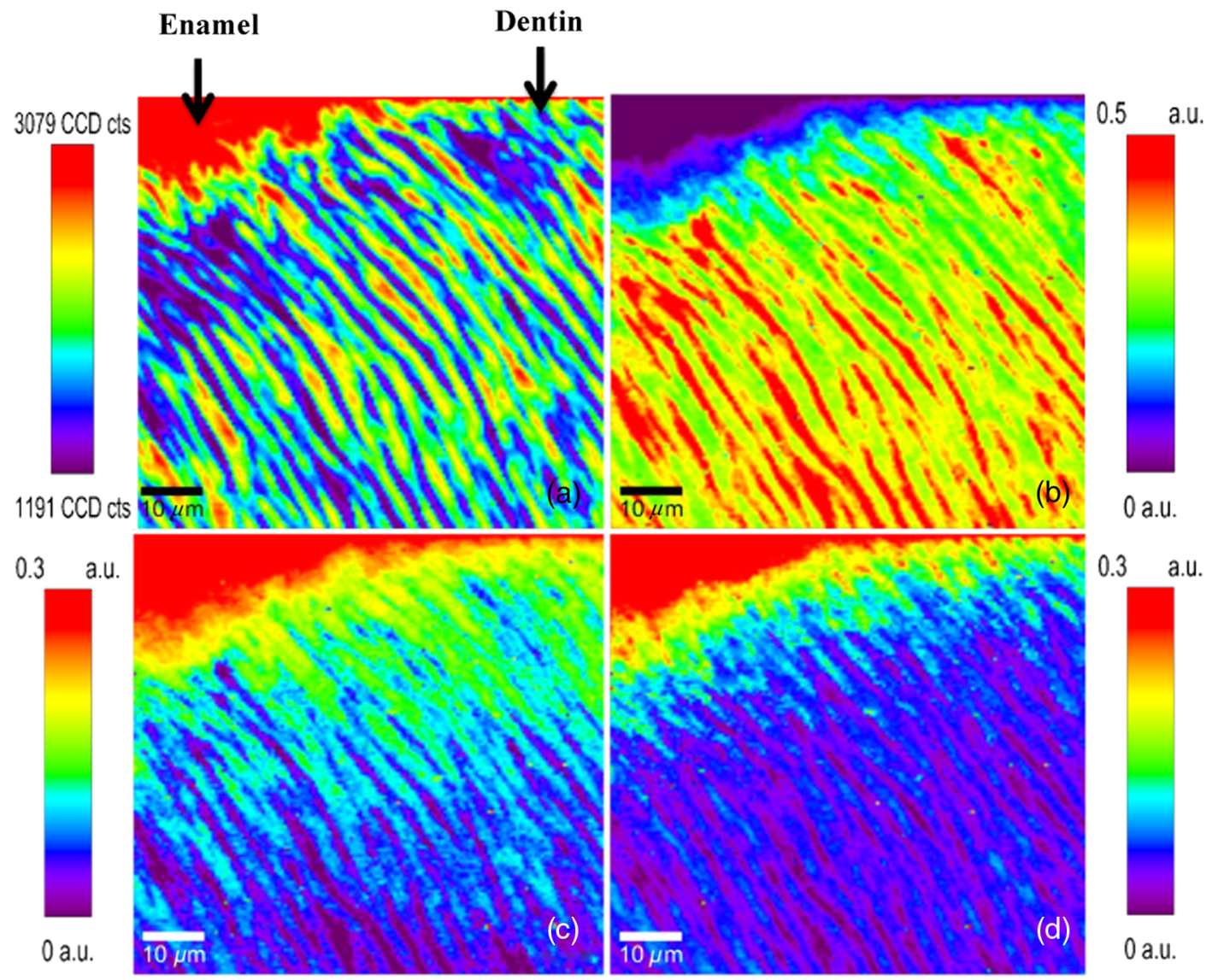

Fig. 3 Raman image of DEJ: (a) Raman intensities of phosphate $\nu_{1} \mathrm{PO}_{4}^{3-}$ peak, (b) collagen crosslink based on intensity ratios of $1660 / 1690 \mathrm{~cm}^{-1}$ peaks, (c) crystallinity calculated from intensity ratios at $960 / 950 \mathrm{~cm}^{-1}$, (d) crystallinity based on the FWHM ${ }^{-1}$ of $\nu_{1}$ phosphate peak in Figs. 3(a)-3(d) with the same LUT: purple represents no signal and red represents the highest intensity.

The phosphate content is high in enamel [left part of Fig. 4(a)] and it decreases during the transition to dentin [right part of Fig. 4(a)]. This transition zone exhibits gradual color differences, which show the decrease of the phosphate peak and some molecular variations as an enamel spindle that appears green. A distinct line appears (dark purple) corresponding to localized rarefaction of phosphate. In the DEJ where in the direction of enamel, the phosphate content increases (green to yellow) and in the direction on the dentin, it decreases to achieve the lowest intensity in the dentinal tubules (linear dark purple). Using the $\mathrm{CH}$ peak to reconstruct an organic image of the DEJ [Fig. 4(b)], enamel appears to be homogeneous with very low to no $\mathrm{CH}$ signal while the collagen crosslink ratio image of the amide I sub-bands [Fig. 4(c)] exhibits heterogamous pattern of the same area (purple to green hues) with traces of collagen crosslinks (red hues) persist along the DEJ. The dentinal tubules are more visible according to the $\mathrm{CH}$ peak representative image [Fig. 4(b)] than in the collagen crosslink image [Fig. 4(c)].

The crystallinity of the scanned area is presented in Fig. 4(d) using the intensity ratio $960 / 950 \mathrm{~cm}^{-1}$. Crystallinity in enamel is maximum and decreases in the dentin because of the wellarranged hydroxyapatite in enamel. The rectangle drawn in Figs. 4(a) -4 (d) is $80 \mu \mathrm{m}$ long and $10 \mu \mathrm{m}$ width. The centered line represents the average spectra (from enamel to dentin) in Fig. 4(e); each point of the line spectrum is the average of 30 spectra. Four premolars were used, and different zones (buccal surface on buccolingual cross-section) were scanned for each sample. Totally, 16 areas were scanned by Raman microscopy, and they were statistically analyzed. An average of $45 \mu \mathrm{m}$ (SD $9 \mu \mathrm{m})$ for the transition zone is obtained. The organic content (red and black spectra) decreases gradually and then abruptly in the small frame between the position from 30 to $45 \mu \mathrm{m}$. In this same area, mineral (blue and green spectra) content increases in the same way. The rate of changes (tangent lines) for four parameters is measured. These parameters exhibit major changes and cross each other in a short window about 12 to $15 \mu \mathrm{m}$ [between position 35 to $48 \mu \mathrm{m}$ on Fig. 4(e)]. The transition zone is estimated around $50 \mu \mathrm{m}$. To select this zone, even slight changes in intensity are considered. This estimation is the width where mineral and organic intensities vary. This DEJ width estimation is wider that those found in previous studies. ${ }^{16-18}$ It is due to the high spatial resolution improved using our imaging technique, which provides more precise information to observe the changes. Crystallinity decreases slowly from the enamel border to half of the transition zone and then drops down. Phosphate signal follows the same pattern but with a huge decrease from the half point. On the contrary, the collagen crosslink signal, from enamel border to dentine, highly increases with similar pattern of the phosphate reduction but varies again inside the transition zone over $10 \mu \mathrm{m}$ as the phosphate signal but with highest intensity. Organic signal also increases, from the enamel border to dentin and reaches a peak, $10 \mu \mathrm{m}$ close to the end of the transition zone.

In detail, Fig. 5 presents two representative crystallinity images of the DEJ. Figure 5(a) uses the intensity of ratios at 


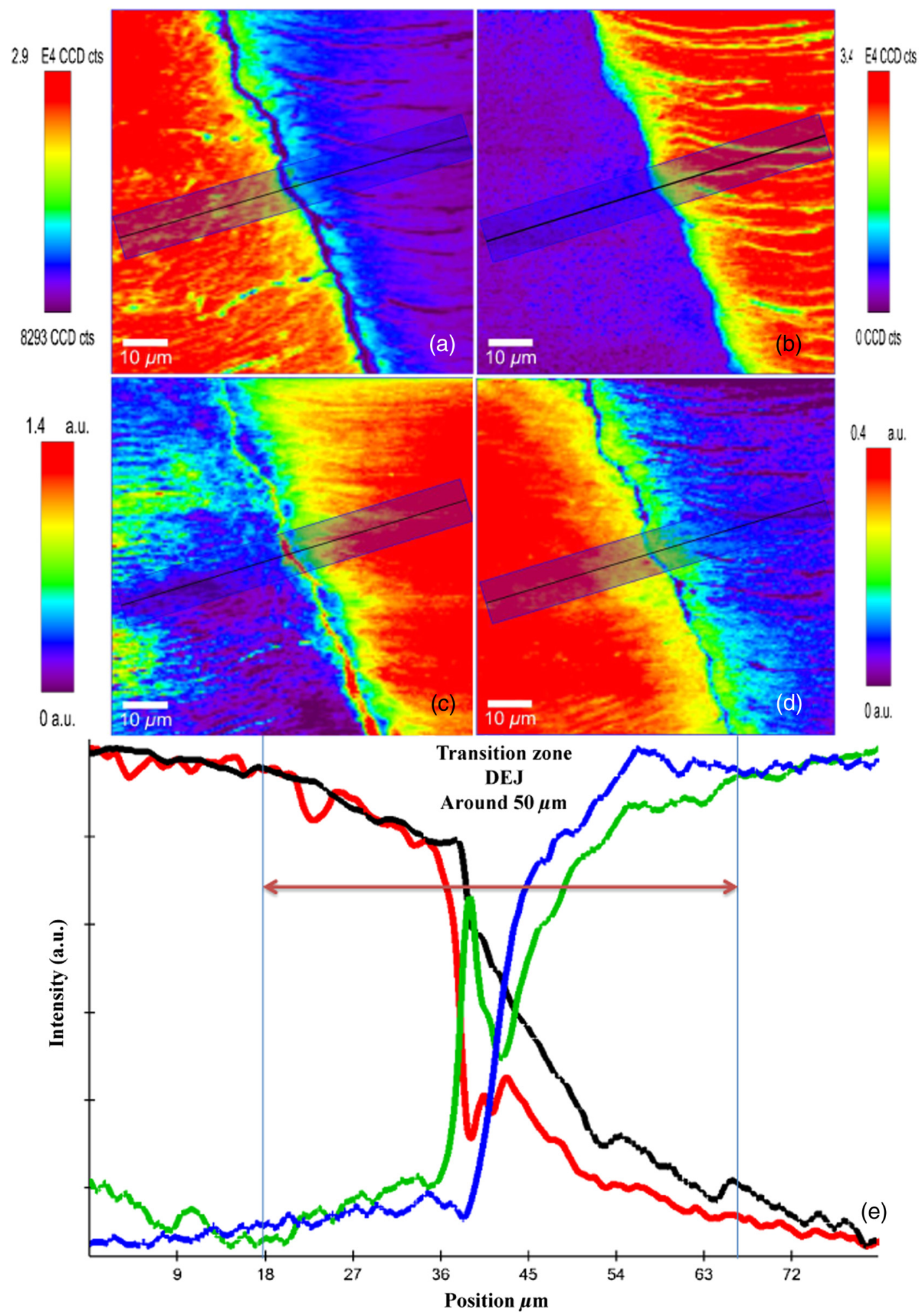

Fig. 4 Reconstructed Raman map of (a) phosphate peak intensity $\left(\nu_{1} \mathrm{PO}_{4}^{3-}, 960 \mathrm{~cm}^{-1}\right)$, (b) organic $\mathrm{CH}$ peak intensity $\left(1450 \mathrm{~cm}^{-1}\right.$ ), (c) collagen crosslink (ratios at 1660/1690 $\mathrm{cm}^{-1}$ ), and (d) crystallinity (ratios at $960 / 950 \mathrm{~cm}^{-1}$ ). Enamel is in left half of all the figures and dentin is in the right half of all the figures. (e) Relative variation across the DEJ in the rectangle drawn (a-d): phosphate peak intensity $\left(960 \mathrm{~cm}^{-1}\right.$; red), organic $\mathrm{CH}, 1450 \mathrm{~cm}^{-1}$ peak intensity (blue), collagen cross-link ratios (green,) and crystallinity (black). The transition zone where changes occur is marked with two vertical blue lines. Enamel is in left half of the graph and dentin in the right half of the graph. 

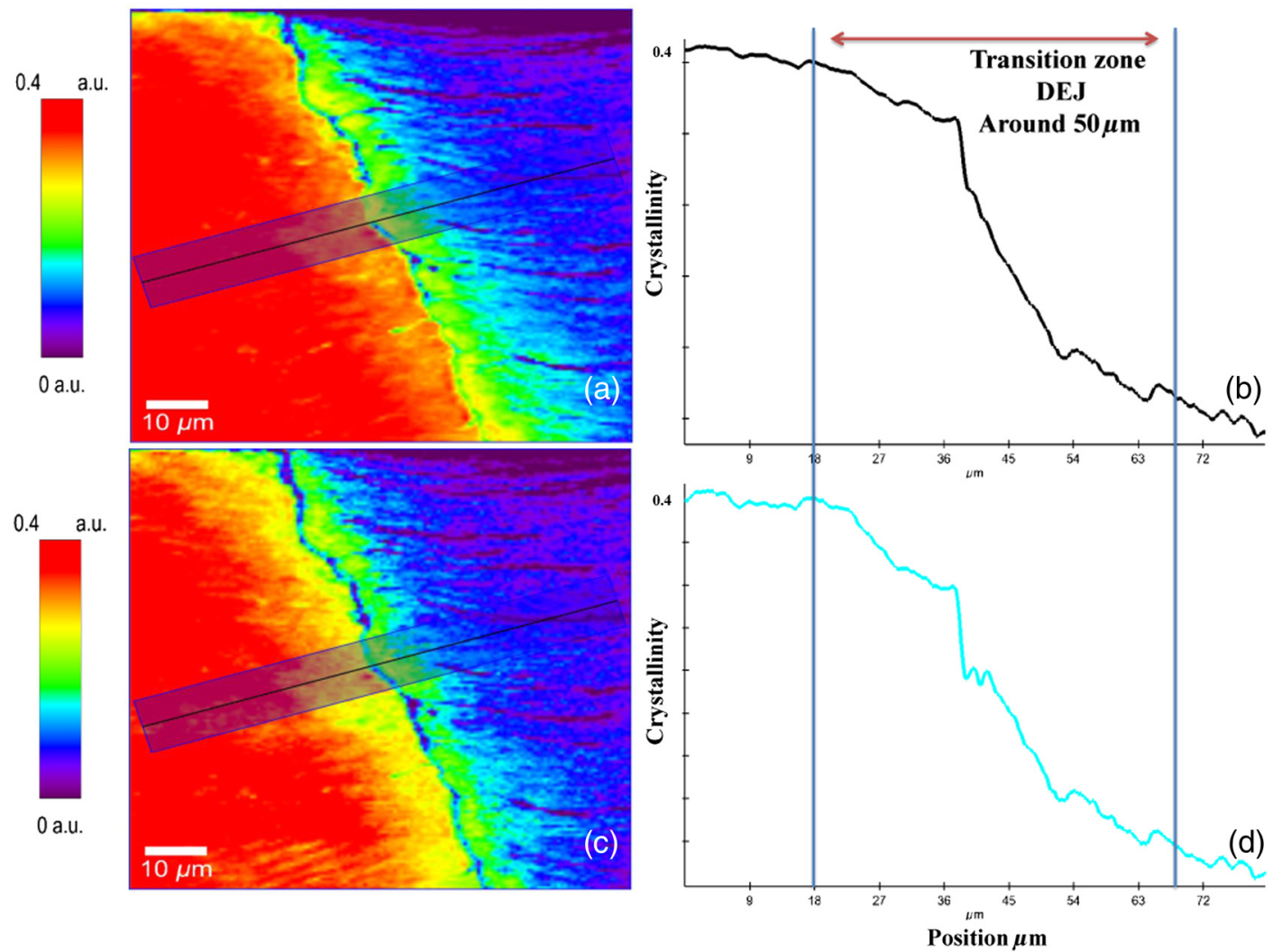

Fig. 5 Crystallinity image (a) ratios at $960 / 950 \mathrm{~cm}^{-1}$ (black spectrum), (b) black spectrum: variation of crystallinity value (method ratios at $960 / 950 \mathrm{~cm}^{-1}$ ) along the solid line in blue rectangle starting from enamel ending in dentin, marking the transition zone with two vertical lines, (c) crystallinity of the $\mathrm{FWHM}^{-1}$ of phosphate peak at $960 \mathrm{~cm}^{-1}$, (d) cyan spectrum: variation of crystallinity value (FWHM ${ }^{-1}$ of phosphate peak at $960 \mathrm{~cm}^{-1}$ ) along the solid line in blue rectangle starting from enamel ending in dentin, marking the transition zone. LUT: purple represents no signal and red represents the highest intensity.

960/950 $\mathrm{cm}^{-1}$ and Fig. 5(c) uses the $\mathrm{FWHM}^{-1}$ (image and average spectrum along the rectangle). Each pixel in parts Figs. 5(a) and 5(c) represents a value for crystallinity with a false color. LUT could help to find the value for each pixel. The graphs in Figs. 5(b) and 5(d) show the change of crystallinity during the passage from enamel to dentin on the dark solid line. The transition zone of DEJ is marked with two vertical lines. This zone shows the change in crystallinity. The first method exhibits an abrupt decrease of crystallinity in the transition zone, whereas the second is more gradual. In general, these two methods provide the same pattern of crystallinity at DEJ.

Figure 6 is a close-up that illustrates the high-spatial resolution imaging of the DEJ with the confocal Raman microscope. Figure 6(a) presents collagen crosslink image by calculation of the ratio of two selected peaks in the amide I band (1660 and $1690 \mathrm{~cm}^{-1}$ ). This collagen crosslink image displays a red

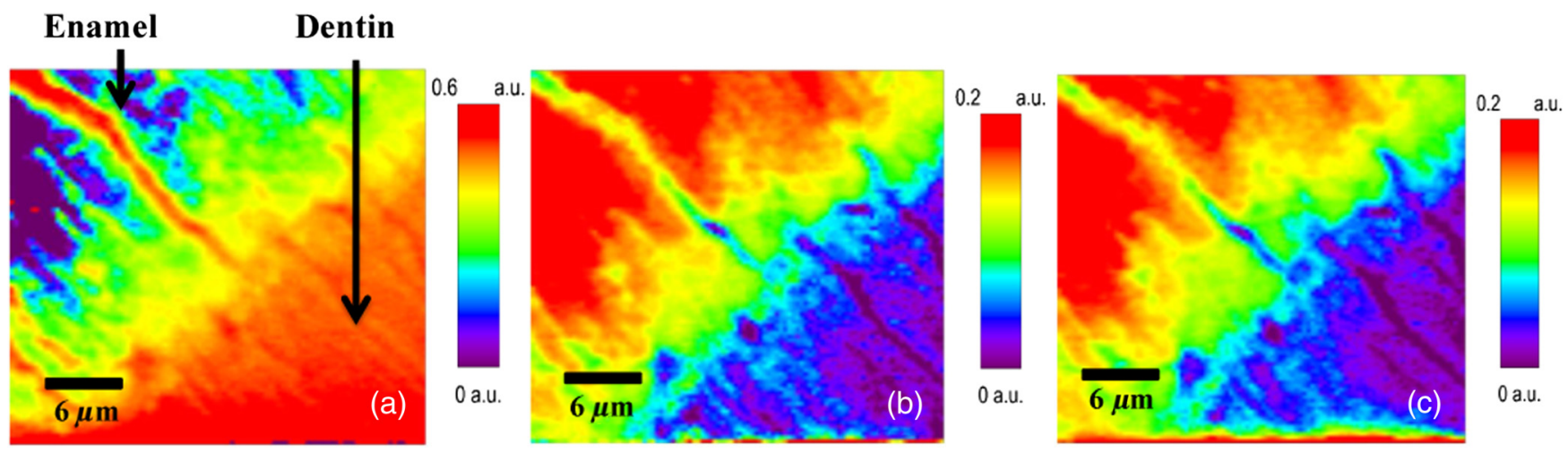

Fig. 6 Representative images of the DEJ: (a) collagen crosslink based on intensity ratios of $1660 / 1690 \mathrm{~cm}^{-1}$, (b) crystallinity of the scanned area based on ratio of $960 / 950 \mathrm{~cm}^{-1}$ peaks), and (c) crystallinity based on intensity of the $\mathrm{FWHM}^{-1}$ of phosphate $\nu_{1}$ mode. LUT: purple represents no signal and red represents the highest intensity 
dentin, which implies the greatest collagen crosslink contents compared to the enamel, where the hues vary gradually from green (average contents) to cyan-blue (low contents) and then dark purple (no collagen crosslink). As explained before, long and broad cracks can be found in enamel as enamel tufts. An enamel tuft is visible: it contains organic material (red: high collagen crosslink) and it seems to be an extension of a dentinal tubule.

In Figs. 6(b) and 6(c), crystallinity of the scanned area is presented. In both images, dentin shows low to absent crystallinity intensities. The inner of the dentinal tubules (organic matrix) has no crystallinity intensity (dark purple) while the enamel tuft shows average crystallinity intensity (green) due its hypocalcified state. Compared to the previous sample, the collagen crosslink and crystallinity is lower. The reason might be the patient age. This claim needs more studies.

\section{Discussion}

DEJ has the property to integrate two dissimilar hard tissues to manage masticatory loading. The decrease of $960 \mathrm{~cm}^{-1}$ phosphate peak intensity was noticed in the inner layer of enamel, which corresponds hypomineralization related to lower hardness and stiffness that helps the integration of two dissimilar tissues. $^{24,25}$

Collagen crosslink is represented by covalent bonds, which provide the stability to the collagen network. The most reported collagen band is amide I. In this spectral zone, the most important bands are 1660 and $1690 \mathrm{~cm}^{-1}$, which belong to $\nu(\mathrm{C}=\mathrm{O})$ component and immature cross-links relatively. The increase in immature crosslink would increase the collagen cross-link. ${ }^{21,26,27}$

In the present study, the collagen crosslink is shown by the reconstruction Raman images for the two selected peaks in the amide I band (1660 and $\left.1690 \mathrm{~cm}^{-1}\right)$. Apparently, the area with high phosphate content shows low collagen crosslink. It seems that the collagen fibers in the DEJ have difficulty to be mineralized. In fact, inside tubules, phosphate concentration is lower, where collagen fibers are more present. The Raman peak at $1660 \mathrm{~cm}^{-1}$ corresponds to a pyridinoline, trivalent, nonreducible crosslink peptide. The amide I, sub-band at $1690 \mathrm{~cm}^{-1}$, belongs to dehydrodihydroxylysinonoreleucine, which is an immature, divalent, reducible crosslink. The peak component at $1690 \mathrm{~cm}^{-1}$ decreases with collagen maturity and age, whereas the component at $1660 \mathrm{~cm}^{-1}$ increases. This is probably caused by the immature divalent crosslinks turning into mature trivalent crosslinks. The crosslink measures perturbation of the secondary structure of the collagen. ${ }^{12}$

Less mineral in enamel at the DEJ, which shows lower hardness of enamel, will guaranty the compatibility of mechanical property at that zone. As the dentin has lower hardness, enamel needs to be adapted slowly to mechanical changes. Therefore, lower hardness is a direct result of less mineral in enamel near DEJ. In return, the residual protein will replace the mineral for the zone close to DEJ. ${ }^{28}$

The sharp peak in green curve in Fig. 4(e) shows the protein residues at the junction, which disappears in few microns. At the same position, a drastic loss of mineral is observed. Raman intensity images for two amide peaks were plotted. No significant amide signal was observed in enamel, although it cannot be excluded. Previous studies reported low protein content in enamel. $^{6,25,29,30}$

Crystallinity represents the stoichiometric perfection of crystal apatite lattice and crystal size. It increases with more perfect crystals with less substitution and larger crystals. Carbonate substitution with longer crystals causes broadening of phosphate band and reduction in crystallinity. Crystallinity refers to the maturation of the calcium phosphate of the dental hard tissues. As mentioned before, the inverse FWHM of Raman peak of phosphate at $960 \mathrm{~cm}^{-1}\left(\nu_{1} \mathrm{PO}_{4}^{3-}\right.$, first vibrational mode) represents the mineral crystallinity. The narrow peak width shows the higher mineral crystallinity. Generally, the crystallinity of enamel hydroxyapatite (HAP) is higher than the dentin. It means that HAP in enamel is regularly arranged. When this arrangement is interrupted, defects like enamel tufts appear and our study here described it with a unique approach using mathematical calculation to evaluate crystallinity. The results show two different methods to calculate the crystallinity on the DEJ area. The two methods present the maximum crystallinity in enamel compared to dentin, which shows more arranged HAP. The crystallinity of DEJ, calculated by $\mathrm{FWHM}^{-1}$ of phosphate peak at $960 \mathrm{~cm}^{-1}$, is calculated using Witec plus software. The second method gives the crystallinity in the same area with the ratio of two phosphate peaks $\left(960 \mathrm{~cm}^{-1}\right.$ over $\left.950 \mathrm{~cm}^{-1}\right)$ overlapped. It shows the same pattern for the crystallinity in DEJ. In the results, the dentin tubules are visible as well as the texture of the enamel and the DEJ area is clearly distinguishable.

In the present study, the transition zone of the DEJ that was estimated around $50 \mu \mathrm{m}$. This DEJ width estimation is wider that those found in previous studies. ${ }^{16-18}$ It is based on the overlap of different Raman peaks intensities of mineral and organic content [Fig. 4(e)] that cross each other at a very specific area of $50 \mu \mathrm{m}$. If sharp slope is considered, then a smaller area will be defined as DEJ. Taking into account small changes - phosphate, organic, collagen crosslink, crystallinity intensities - a transition zone of average $45 \mu \mathrm{m}$ is defined.

\section{Conclusion}

Confocal Raman microscopy proved to be useful in obtaining chemical and structural information, and collagen cross-link and crystallinity map of DEJ. In terms of crystallinity between the two suggested methods, $\mathrm{FWMH}^{-1}$ and phosphate peak ratio, both gave relevant information. In this study, amide I and phosphate sub-band ratios to obtain collagen cross-link and crystallinity are measured. These ratios could provide information about sample with minimum sample preparation and high spatial resolution. By plotting different Raman peaks ratio, a quantitative analysis is provided. Our results demonstrate that confocal Raman microscopy is a powerful label-free and noninvasive imaging technique to be applied to study chemical structure of DEJ. However, to obtain quantitative information, appropriate data analysis methods should be applied to the measured raw Raman spectra. Postmeasurement data analysis gives us the possibility to obtain more information about maturation and mechanism of growth of calcium phosphate or crystallinity and collagen crosslink. Based on the results provided here, the next step would be applying these calculations and methods to compare the effect of aging on crystallinity and collagen crosslink. Collagen fibrils and the polymeric chains have a major role in the stability of the adhesive interface. It is especially important to better understand the action of collagen cross-linkers on dentin bonding (e.g., proanthocyanidin, UVA-riboflavin), which minimized the time degradation without jeopardizing the adhesive polymerization, as well as reducing the collagenolytic activity of matrix metalloproteinases. ${ }^{31,32}$ The mentioned information could help dentists for efficient diagnosis and improve dental 
materials with dissimilar mechanical properties in operative dentistry.

\section{Disclosures}

No conflicts of interest, financial or otherwise, are declared by the authors.

\section{References}

1. J. Meyer et al., "Initial aspects of mineralization at the dentino-enamel junction in embryonic mouse incisor in vivo and in vitro: a tem comparative study," In Vitro Cell. Dev. Biol. Anim. 35(3), 159-168 (1999).

2. P. Houllé et al., "High resolution electron microscopy: structure and growth mechanisms of human dentin crystals," J. Dent. Res. 76(4), 895-904 (1997)

3. E. Beniash et al., "A transmission electron microscope study using vitrified ice sections of predentin: structural changes in the dentin collagenous matrix prior to mineralization," J. Struct. Biol. 132(3), 212-225 (2000)

4. M. Goldberg et al., "Dentin: structure, composition and mineralization," Front. Biosci. 3, 711-735 (2011).

5. S. N. White et al., "The dentino-enamel junction is a broad transitional zone uniting dissimilar bioceramic composites," J. Am. Ceram. Soc. 83(1), 238-240 (2000).

6. T. Cloitre et al., "Multiphoton imaging of the dentine-enamel junction," J. Biophotonics 6(4), 330-337 (2013).

7. A. R. Ten Cat, Oral Histology: Development, Structure and Function, Mosby Inc., St. Louis (1994).

8. C. M. P. Vidal et al., "Mimicking the hierarchical functions of dentin collagen cross-links with plant derived phenols and phenolic acids," Langmuir 30(49), 14887-14893 (2014).

9. D. R. Eyre, M. A. Weis, and J. J. Wu, "Advances in collagen cross-link analysis," Methods 45(1), 65-74 (2008).

10. Y. Liu et al., "Molecular weight and galloylation affect grape seed extract constituents ability to cross-link dentin collagen in clinically relevant time," Dent. Mater. 31(7), 814-821 (2015).

11. R. Z. LeGeros, "Calcium phosphates in demineralization/remineralization processes," J. Clin. Dent. 10(2), 65-73 (1999).

12. M. A. Alebrahim, "ATR-FTIR and Raman imaging to study permanent and primary teeth from different laces and ages," Dissertation, Jena University (2013).

13. M. L. Paine et al., "Regulated gene expression dictates enamel structure and tooth function," Matrix Biol. 20(5-6), 273-292 (2001).

14. P. Fratzl, "Collagen: structure and mechanics, an introduction," in Collagen: Structure and Mechanics, pp. 1-13, Springer, Boston (2008).

15. J. K. Avery and D. J. Chiego, Essentials of Oral Histology and Embryology, 3rd ed., Mosby, St. Louis (2010).
16. S. Habelitz et al., "The functional width of the dentino-enamel junction determined by AFM-based nanoscratching," J. Struct. Biol. 135(3), 294-301 (2001)

17. H. Fong et al., "Nano-mechanical properties profiles across dentinenamel junction of human incisor teeth," Mater. Sci. Eng. C 7(2), 119-128 (2000).

18. G. W. Marshall et al., "Mechanical properties of the dentinoenamel junction: AFM studies of nanohardness, elastic modulus, and fracture," J. Biomed. Mater. Res. 54(1), 87-95 (2001).

19. A. Desoutter et al., "Structure and chemical composition of the dentinenamel junction analyzed by confocal Raman microscopy," Proc. SPIE 8929, 892907 (2014).

20. C. Xu et al., "Chemical/molecular structure of the dentin-enamel junction is dependent on the intratooth location," Calcif. Tissue Int. 84(3), 221-228 (2009).

21. H. Salehi et al., "Functional mapping of human sound and carious enamel and dentin with Raman spectroscopy," J. Biophotonics 6(10), 765-774 (2013)

22. H. Salehi et al., "Confocal Raman microscopy to monitor extracellular matrix during dental pulp stem cells differentiation," J. Biomed. Opt. 20(7), 076013 (2015).

23. R. L. Frost et al., "Structural characterization and vibrational spectroscopy of the arsenate mineral wendwilsonite," Spectrochim. Acta Part A 118, 737-743 (2014).

24. L. C. Cuy et al., "Nanoindentation mapping of the mechanical properties of human molar tooth enamel," Arch. Oral Biol. 47(4), 281-291 (2002).

25. S. Habelitz, "Materials engineering by ameloblasts," J. Dent. Res. 94(6), 759-767 (2015).

26. G. S. Mandair and M. D. Morris, "Contributions of Raman spectroscopy to the understanding of bone strength," Bonekey Rep. 4, 620 (2015).

27. E. P. Paschalis et al., "Spectroscopic characterization of collagen crosslinks in bone," J. Bone Miner. Res. 16(10), 1821-1828 (2001).

28. L. Moradian-Oldak, "Protein-mediated enamel mineralization," Front. Biosci. 17, 1996-2023 (2012).

29. J. D. McGuire et al., "Type IV collagen is a novel DEJ biomarker that is reduced by radiotherapy," J. Dent. Res. 93(10), 1028-1034 (2014).

30. V. Dusevich et al., "Identification of a protein-containing enamel matrix layer which bridges with the dentine-enamel junction of adult human teeth," Arch. Oral Biol. 57(12), 1585-1594 (2012).

31. C. Xu and Y. Wang, "Collagen cross linking increases its biodegradation resistance in wet dentin bonding," J. Adhes. Dent. 14(1), 11-18 (2012).

32. V. Hass et al., "Collagen cross-linkers on dentin bonding: stability of the adhesive interfaces, degree of conversion of the adhesive, cytotoxicity and in situ MMP inhibition," Dent. Mater. 32(6), 732-741 (2015).

Biographies for the authors are not available. 